Western University

Scholarship@Western

Mechanical and Materials Engineering

Mechanical and Materials Engineering

Publications

Department

2016

\title{
Forced convective heat transfer on a horizontal circular cylinder due to multiple impinging circular jets
}

Christopher M. Csernyei

The University of Western Ontario

Anthony G. Straatman

uwo, agstraat@uwo.ca

Follow this and additional works at: https://ir.lib.uwo.ca/mechanicalpub

Part of the Materials Science and Engineering Commons, and the Mechanical Engineering Commons

\section{Citation of this paper:}

Csernyei, Christopher M. and Straatman, Anthony G., "Forced convective heat transfer on a horizontal circular cylinder due to multiple impinging circular jets" (2016). Mechanical and Materials Engineering Publications. 20.

https://ir.lib.uwo.ca/mechanicalpub/20 
Research Paper

\title{
Forced convective heat transfer on a horizontal circular cylinder due to multiple impinging circular jets
}

\author{
Christopher Csernyei, Anthony G. Straatman* \\ Department of Mechanical \& Materials Engineering, Western University, London, Ontario N6A 5B9, Canada
}

\section{H I G H L I G H T S}

- Consideration of bank of impinging circular jets with high d/D ratio.

- Results based on more than 100 computational fluid dynamics cases.

- For largest jets, heat transfer decreases with movement away from cylinder axis.

- For smaller jet, heat transfer increases with movement away from cylinder.

- Correlation concisely quantifies heat transfer for parametric range considered.

\section{A R T I C L E I N F O}

\section{Article history:}

Received 22 February 2016

Revised 12 May 2016

Accepted 27 May 2016

Available online 28 May 2016

\section{Keywords}

Impinging jet

Convection

CFD

Rotary kiln

\begin{abstract}
A B S T R A C T
A computational study is undertaken to investigate convective heat transfer on a horizontal cylinder due to a bank of vertically oriented circular jets. The importance of this study stems from the real-world application of convective cooling of rotary cement kilns by the use of large axial fans. A computational model is developed which considers one spatially-periodic section of the domain, and solutions of the conservation equations combined with an appropriate two-equation turbulence model are obtained using the commercial software Fluent ${ }^{\mathrm{TM}}$. The computational model is validated by a comparison to previous studies of a single circular jet impinging on a cylinder. A parametric study is presented which considers the impact on average heat transfer due to: jet-to-cylinder spacing $(y / d)$, axial jet spacing $(z / d)$, jet-to-cylinder diameter ratio $(d / D)$, jet offset from the axial centerline $(x / d)$, and jet Reynolds number. Results of the parametric study show that for jet-to-cylinder ratios of $d / D \geqslant 0.23$, that movement away from the cylinder axis, increased axial spacing and lateral offset all lead to degradation of heat transfer from the cylinder. For $d / D=0.15$, similar degradation occurs, but in part of the parametric space studied, increased distance from the cylinder and lateral offset leads to enhancements of the heat transfer. The complete set of results is presented as a correlation wherein the influence of all parameters studied are included as power-law corrections to the average Nusselt number.
\end{abstract}

(c) 2016 Elsevier Ltd. All rights reserved.

\section{Introduction}

Convective cooling of large cylinders by banks of fans has many applications in the process industry. A pertinent example is in the cement industry where large (4-5 $\mathrm{m}$ in diameter and $50-100 \mathrm{~m}$ in length) rotary kilns are utilized for the production of cement clinker. A schematic showing the layout of a typical rotary cement kiln is given in Fig. 1. Tilted at an angle of $2-5^{\circ}$ with respect to horizontal, and operating at a slow rotational speed of approximately 1-5 rpm, the raw feed (typically limestone, silica, aluminum and iron oxide, Mujumdar and Ranade [1]) travels through the

\footnotetext{
* Corresponding author.

E-mail address: astraatman@eng.uwo.ca (A.G. Straatman).
}

cylindrical kiln due to gravity/tumbling. The kiln itself is comprised of a steel shell for strength and rigidity, lined on the inner surface with refractory brick to enhance the thermal resistance, and isolate the steel shell from the high temperature process taking place inside. Raw feed enters the kiln at one end, and fuel (petroleum coke), which is combusted in a burner located approximately $1 \mathrm{~m}$ into the kiln, enters at the opposite end. Near the burner, the temperature of the exhaust gases can reach upwards of $2200 \mathrm{~K}$, and the reacting raw material can reach as high as $1900 \mathrm{~K}$. The solids begin to melt in this region allowing for the required solid-liquid reactions to occur. As this liquid mixture re-solidifies, tiny balls referred to as clinker are formed, as well as a thin coating layer adjacent to the inner refractory wall of the kiln. This process is essential to the formation of clinker, and requires significant heat 


$\begin{array}{ll}\text { Nomenclature } \\ d & \text { jet diameter, m } \\ D & \text { cylinder diameter, m } \\ y & \text { jet-to-cylinder distance, } \mathrm{m} \\ z & \text { axial distance, m } \\ x & \text { jet offset from cylinder axis, m } \\ \operatorname{Re}_{d} & \text { jet Reynolds number } \\ \operatorname{Re}_{D} & \text { cylinder Reynolds number } \\ k & \text { turbulent kinetic energy, } \mathrm{m}^{2} / \mathrm{s}^{2}\end{array}$

$y^{+} \quad$ non-dimensional wall distance

$V_{J} \quad$ airspeed of fluid at jet exit, $\mathrm{m} / \mathrm{s}$

$h_{\text {ave }} \quad$ average heat transfer coefficient, $\mathrm{W} / \mathrm{m}^{2} \mathrm{~K}$

$\mathrm{Nu}_{\text {ave }}$ average Nusselt number

$k_{f} \quad$ thermal conductivity of fluid, $\mathrm{W} / \mathrm{m} \mathrm{K}$

$\varepsilon \quad$ turbulent dissipation rate, $\mathrm{m}^{2} / \mathrm{s}^{2}$

$v \quad$ kinematic viscosity, $\mathrm{m}^{2} / \mathrm{s}$ removal through the kiln wall to occur. To facilitate heat transfer through the kiln wall, banks of large kiln shell cooling fans are often used in the burning region of the kiln.

Positioning of the cooling fans requires consideration of the process occurring in the kiln, and the path that the heat takes to reach the outer wall of the kiln. Fig. 2 shows that the particle bed inside the kiln is skewed to one side, due to the slow rotation of the kiln, and that the particle bed is in contact with the only 10$15 \%$ of the refractory material at a time. While this produces a local hot-spot on the inner refractory surface, this hot spot is in continuous motion due to the rotation of the kiln. A report by Mastorakos et al. [2] indicates that the internal surface temperature can vary by $\pm 50 \mathrm{~K}$ from an average temperature value. In their report, internal heat transfer is described as a "regenerative" process, wherein the refractory material receives heat when exposed to the flame and internal freeboard gas inside of the kiln, and then supplies heat to the tumbling raw material bed inside of the kiln as it passes underneath. In the "burning" region, where the fans are typically located, a melt forms inside of the kiln, which solidifies over the refractory brick forming the thin coating layer mentioned previously. The thermal conductivity of this coating layer mitigates conductive heat transfer through the wall and can result in a kiln shell temperature drop of $200-250 \mathrm{~K}$ [1]. Then, as heat flows past the coating layer through the refractory brick and kiln shell, the tangential variations are smoothed resulting in total tangential differences much less than those on the inside, which is why the external surface is approximated as being isothermal. Despite this fact, many production facilities offset the fans so that they are directed more closely at the tumbling particle bed, which may or may not enhance the overall heat transfer from the kiln. The purpose of this study is to consider a bank of impinging fans directed at the kiln wall at different distances from the kiln shell, different

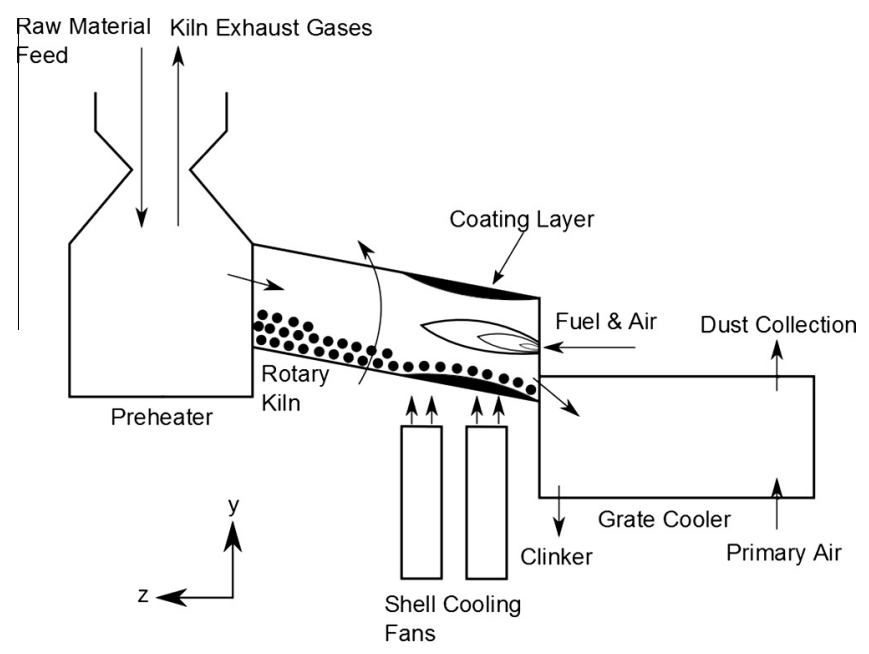

Fig. 1. Schematic of a rotary cement kiln. axial separation, and different lateral offsets from the kiln centerline to quantify the convective heat transfer and specifically, to understand the degradation/enhancement in heat transfer associated with offsetting the fans away from the cylinder axis. In the interest of generality, the kiln in this study is considered to be a horizontal cylinder and rotation is ignored; as mentioned above, the slow rotation only serves to make the outer surface more nearly isothermal, which is the heating condition used in this study. While there have been many studies that have considered convective heat transfer due to impinging jets and banks of jets, they have been predominantly focused on impingement on a flat plate. The most notable of these are the works of Martin [3], Jambunathan et al. [4], Zuckerman and Lior [5] and Yule [6]. In addition, the effect of turbulence on heat transfer by an impinging slot-jet on a flat plate was studied by Gardon and Akfirat [7]. Far fewer investigations of jet impingement on a circular cylinder exist in open literature, especially for cases where the jet to cylinder diameter is large.

Several studies involving impingement on a cylinder relate to impingement from slotted jets that are oriented with the axis of the cylinder. Olsson et al. [8] studied the effect of a single slot jet impinging on a single cylinder for operational parameters such as jet-to-cylinder distance, and cylinder curvature for jet Reynolds numbers (based on jet exit velocity and slot width) ranging from 23,000 to 100,000 . It was concluded that average and stagnation Nusselt numbers increase with increasing jet Reynolds number and surface curvature; however, the effect of jet-to-cylinder spacing was not found to be significant. Another study by Olsson et al.

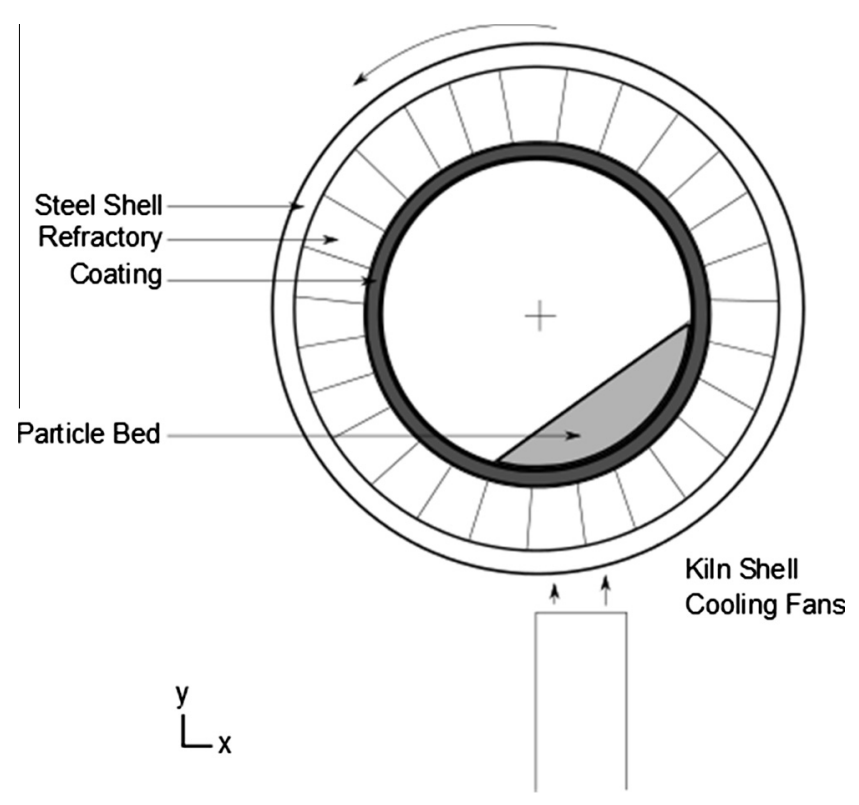

Fig. 2. Cross-section of rotary cement kiln. 
[9] considered multiple slotted jets impinging on multiple cylinders for the same range of parameters as in [10], but including the non-dimensional effect of distance between the jets. Here, they observed that variation of the distance between jets and the opening of the jets were important when trying to achieve higher rates of heat transfer. Jeng et al. [10] studied the effect of a lateral impinging slotted air jet on a rotating cylinder for jet Reynolds numbers in the range $655-60,237$ and for a range of rotational Reynolds numbers from 0 to 7899. They showed that the average Nusselt number increases as the rotational Reynolds number increases and that this effect is reduced for higher jet Reynolds numbers. Another study involving a single slotted jet along the length of a free cylinder was conducted by McDaniel and Webb [11], where it was shown that an optimal nozzle-to-cylinder spacing exists where maximum heat transfer occurs. Gori and Bossi [12,13] also investigated experimentally the effect of slot width to cylinder separation distance for a fixed cylinder diameter $(D)$ and slot to cylinder spacing $(H)$, within ratios of $H / D$ ranging from 2 to 10 for cylinder Reynolds number, $R e_{D}$, between 4000 and 20,000. This study also considered the effect of slot width (S) through the dimensionless ratio of cylinder diameter over slot width, $D / S$, at values of 1,2 , and 4 , and found that the maximum Nusselt number occurs at $H / D=8$ [13]. Sparrow and Alhomoud [14] provide a comprehensive study on the effect of Sherwood number when offsetting a single slot jet from the axis of the cylinder that it is impinging upon. The study showed that offsetting the jet decreased the Sherwood number, and that the effect was greatest for small slot-to-cylinder separation distances. Furthermore, the results were sensitive to modification of the slot width, especially as the offset was increased.

The effect of an array of slotted jets impinging on a cylinder has been investigated experimentally by Nada [15], where a comparison is drawn between a single slot jet aligned with the cylinder axis and multiple slot jets aligned orthogonal to the cylinder axis. Similar to previous studies, the effect jet-to-jet spacing and jet-tocylinder spacing were examined. Again, it was found that there exists an optimal region of $H / S$ where the average Nusselt number reached a maximum. Zuckerman and Lior [16] considered different turbulence models in their numerical study of convective heat transfer from a circular cylinder. Their simulations study the effect of 2-8 slot jets positioned equally-spaced radially around a cylinder for a range of operating parameters and jet Reynolds number from 5000 to 80,000 . The so-called "fountain flow" shape generated from the secondary region of impingement between two adjacent jets was shown to greatly increase the heat transfer from the cylinder. A correlation was proposed to characterize the heat transfer from the cylinder for 2-8 slot jets equally spaced radially around the cylinder.

Existing research on convective heat transfer due to circular impinging jets on a cylinder primarily focuses on single, small circular jets impinging directly on the axis of the cylinder. To this end, Tawfek [17] conducted an experimental investigation of a single circular jet impinging on an isothermally heated brass cylinder. Three different jet diameters and various jet-to-cylinder separation distances were studied for $R e_{j}$ ranging from 3800 to 40,000 . The highest heat transfer was observed at the region of impingement. They also observed that as the jet-to-cylinder spacing increased, stagnation heat transfer rate decreased. Turbulent flow and convective heat transfer over a curved surface was examined by Lee et al. [18]. The jet potential core length was observed to range from $3.1 d$ to $4.2 d$ for the range of jet Reynolds numbers from 11,000 to 50,000 . The stagnation Nusselt number was reported to vary with increasing jet-to-cylinder spacing $(L / d)$ reaching a maximum between $L / d=6-8$. Kumada et al. [19] observed the effect of heat and mass transfer from a circular jet impinging on a cylinder within the potential core. Utilizing a naphthalene sublimation technique, an experimental analysis was performed. One of the conclusions was that flow around a cylinder follows the Coanda effect, which describes the tendency for a fluid to remain attached to a convex surface. A large pressure gradient was observed from the stagnation region at impingement, while at the rear stagnation point, where flow is separated, the pressure coefficient is either zero or positive. Following the Coanda effect, a negative pressure gradient can be observed while the flow remains attached to the convex surface. Wang et al. [20] studied three different cylinderto-jet diameter ratios, $D / D_{j}=0.5,2.0$, and 5.0 for a fixed jet diameter $D_{j}$, and a jet Reynolds number of 20,000 . They concluded that ratios of $D / D_{j} \geqslant 2.0$ follow similar local heat transfer characteristics for circular jets impinging on a flat plate. Wang et al. [21] performed another study focusing on the heat transfer characteristics of an inclined circular jet impinging on cylinder when $D / D_{j}=5.0$, 10.0 for a fixed jet diameter and jet Reynolds number of 20,000. Based on the orientation of the jet to the cylinder, this study served as the closest representation of an offset circular jet impinging on a large cylinder. The jet was inclined from the axial centerline by angles of $0^{\circ}, 15^{\circ}, 30^{\circ}$, and $45^{\circ}$. It was concluded that for the ratio jet spacing to jet diameter of $y / D_{j}=2.0$, an increase in stagnation Nusselt number, as well as local Nusselt number both axially and tangentially was observed on the "downhill" face of the cylinder. This effect was reduced as the $y / D_{j}$ spacing increased. A study performed by Sparrow et al. [22] used a naphthalene sublimation technique to quantify heat transfer from a cylinder in the area of impingement. Correlations for stagnation point Nusselt and Sherwood numbers were derived from experiments conducted in the range of $R e_{j}$ from 4000 to 25,000 and non-dimensional jet-tocylinder spacing $H / d$ from 5 to 15 for three different jet diameters. Peak heat transfer was determined to exist as the jet-to-cylinder spacing decreased, and as the jet diameter decreased. A detailed experimental and computational approach to turbulent impingement of a single cylinder for parameters of jet-to-cylinder spacing from 4 to 16, and the ratio of jet diameter over cylinder diameter, $d / D$, from 0.11 to 0.25 for $R e_{j}$ from 10,000 to 25,000 was performed by Singh et al. [23]. Various different turbulence models were tested for the numerical model. From this study it was concluded that stagnation Nusselt number increases consistently as jet-tocylinder spacing decreases. It was also determined that outside of the region of impingement, modifications to jet-to-cylinder spacing and $d / D$ do not have an impact on heat transfer. Furthermore, regardless of the turbulence model used, stagnation Nusselt number was over-estimated. Lastly, Dong et al. [24] conducted a study on a row of three cylindrical butane/air flame jets impinging on a flat plate. Through the modification of jet-to-jet and jet-tocylinder spacing for values of $(2,5)$ and $(2.6,5,7)$, respectively, they concluded that as the jet-to-cylinder spacing increased, the area-averaged heat flux also increased. Additionally, heat transfer was shown to increase to a peak value before decreasing again with the increase of jet-to-jet spacing.

To the knowledge of the authors, no studies exist in the literature that consider banks of highly turbulent circular jets of large jet-to-cylinder diameter ratio impinging on a circular cylinder at different axial and lateral offsets. The unique nature of this phenomenon within an industrial application is the driving motivation of this study. In this respect, the question that will be answered is whether there is a jet position relative to the cylinder axis that yields optimal heat transfer, or conversely, how much degradation/enhancement in heat transfer is occurring when the bank of jets is not (or cannot) be positioned directly under the cylinder axis. Moreover, is there a jet-to-cylinder distance (in close range) that produces maximum heat transfer? This study presents numerical simulations over a range of jet-to-cylinder ratios, jet-tocylinder distances, and axial and lateral offsets to specifically determine the convective heat transfer performance of the system. 
Parameters have been selected based on those relevant to the cement production industry where such configurations are realized. Validation of the numerical model is done by comparing to the work of previous studies on single impinging jets. A correlation is provided that characterizes the forced convective heat transfer for the entire parametric space considered in terms of relevant dimensionless quantities.

\section{Problem formulation}

A schematic of the horizontal cylinder geometry with the bank of vertical jets considered in this study is shown in Fig. 3. While the dimensions of the cylinder and jet are scaled out in the presentation of results, rotary kilns typically have a diameter in the range 4-5 $\mathrm{m}$ and a length in the range $50-100 \mathrm{~m}$, and these sizes require consideration for determination of a relevant Reynolds number range. The cylinder shown in Fig. 3 is assumed to have a large length-to-diameter $(L / D)$ aspect ratio and the bank of vertical jets is evenly spaced. By these assumptions, only a single repeating segment of the geometry is required in the simulations. The symmetry planes shown in Fig. 3 reduce the geometry substantially by cutting through the centerline of a vertical jet, and the center-plane between two vertical jets. This serves to reduce the grid size and simulation run time by an enormous amount and was done for all simulations. The computational domain for the reduced segment is shown in Fig. 4. The model is shown to have an inner cylin-
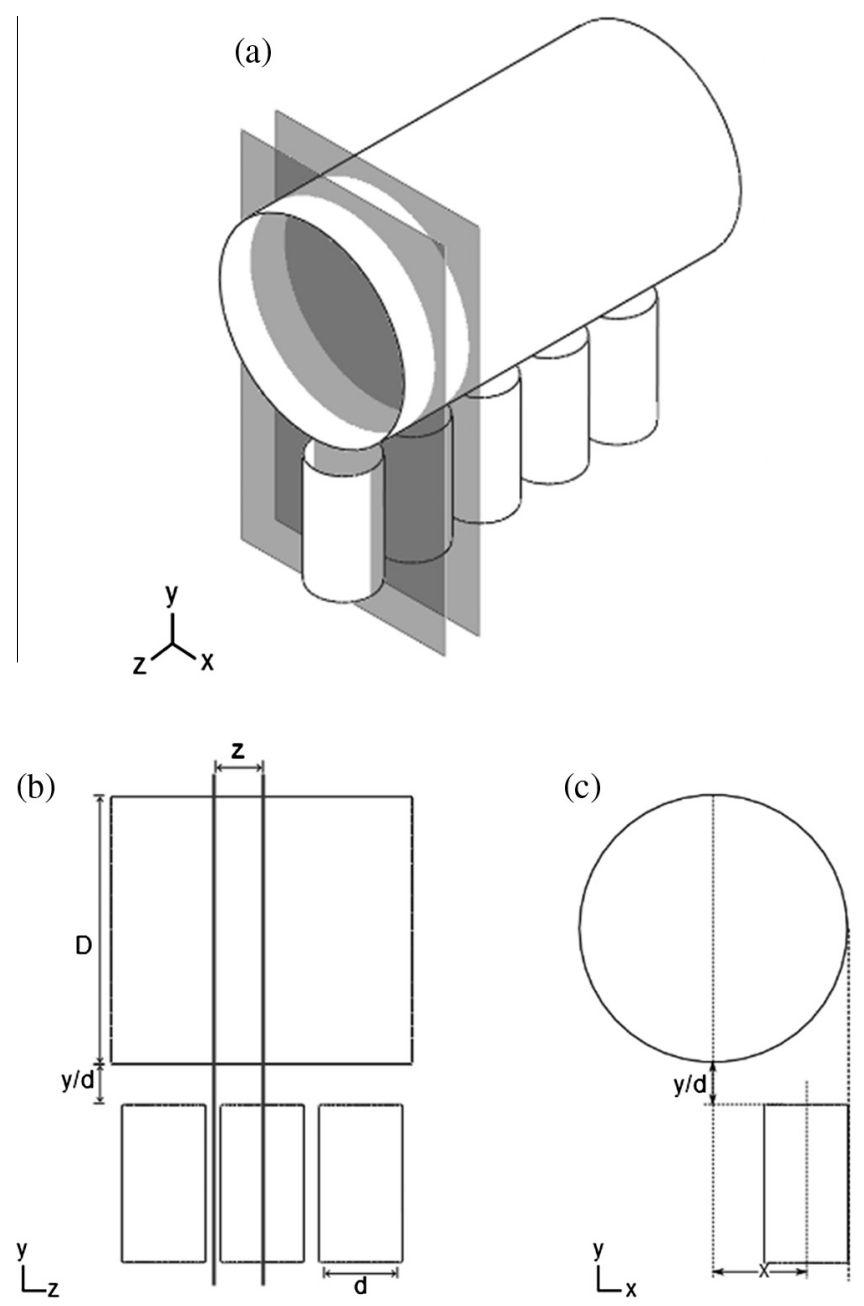

Fig. 3. Geometric model of horizontal cylinder and vertical jet bank. (a) Isometric view, (b) side view and (c) front view.

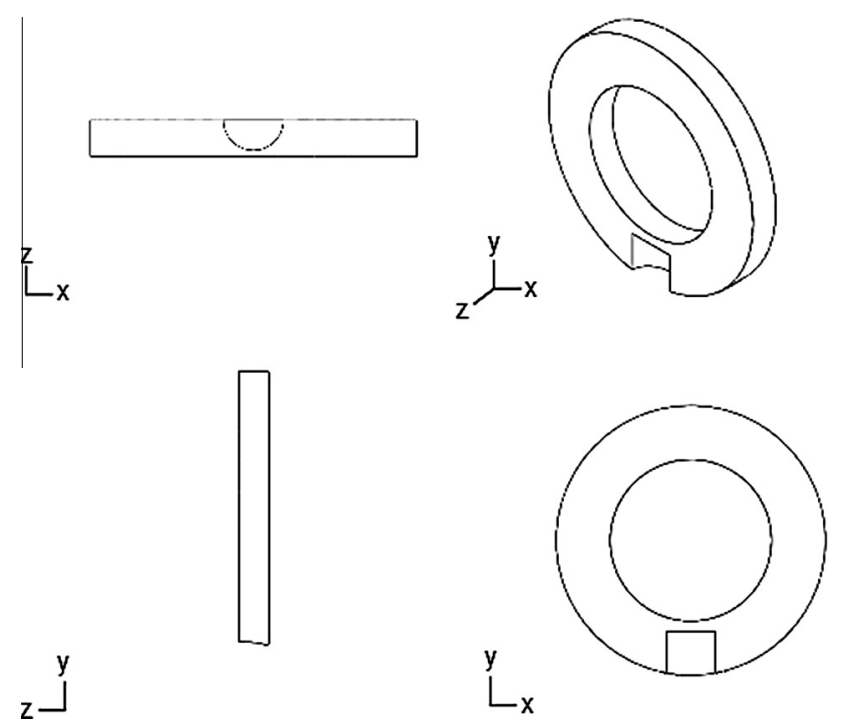

Fig. 4. Reduced geometry as extracted from Fig. 3(a).

der wall, which is assumed isothermal, a circular far-field boundary, which is discussed further in the model validation, and a semi-circular jet inlet, which represents the vertical jet bank. Using this model, the jet spacing $(z / d)$ is varied by changing the width between symmetry planes; the jet position relative to the cylinder $(y / d)$ is changed by adjusting the length of the vertical inlet region; and the jet offset $(x / d)$ is adjusted by moving the position of the vertical inlet region away from the axial centerline.

The horizontal cylinder diameter was fixed at a diameter of $D=4.8 \mathrm{~m}$. The non-dimensional jet to cylinder diameter was varied across $d / D=0.15,0.23$, and 0.31 by varying the jet diameter, $d$. The jet-to-cylinder spacing, $y / d$, was varied from 0.21 to 2.14 , by considering physical dimensions of $y=0.305-1.524 \mathrm{~m}$. Each case was then simulated for the same jet-to-jet spacings, $z / d$, which ranged from 1.21 to 3.41. Finally, in terms of jet offset, the vertical jet ranged from being positioned directly beneath the axial centerline of the horizontal cylinder $(x / d=0)$, to being offset to the point where the outer edge of the jet was aligned with the edge of the horizontal cylinder. Since the jet diameter varied in this study, jets with a smaller diameter had higher offset values when positioned at their maximum offset, which is given as:

$\left(\frac{x}{d}\right)_{\max }=(D-d) / 2 d$

\section{Mathematical formulation}

ANSYS FLUENT 13.0 was used to solve the governing equations for mass, momentum, turbulence and energy. The governing equations are as given in the ANSYS FLUENT Theory Guide [25]:

Conservation of mass:

$\frac{\partial \rho}{\delta t}+\nabla \cdot(\rho \vec{v})=S_{m}$

Conservation of momentum:

$\frac{\partial}{\partial t}(\rho \vec{v})+\nabla \cdot(\rho \vec{v} \vec{v})=-\nabla p+\nabla \cdot(\overline{\bar{\tau}})+\rho \vec{g}+\vec{F}$

Conservation of energy:

$\frac{\partial}{\partial t}(\rho E)+\nabla \cdot(\vec{v}(\rho E+p))=\nabla \cdot\left(k_{e f f} \nabla T-\sum_{j} h_{j} \vec{J}_{j}+\left(\overline{\bar{\tau}}_{e f f} \cdot \vec{v}\right)\right)+S_{h}$ 
where the stress tensor in the momentum equation is defined as:

$\overline{\bar{\tau}}=\mu\left[\left(\nabla \vec{v}+\nabla \vec{v}^{T}\right)-\frac{2}{3} \nabla \cdot \vec{v} I\right]$

Since all cases considered are fully-turbulent, selection of a turbulence model is also necessary. To this end, the industrystandard, $k-\varepsilon$ turbulence model was used with standard wall functions. While the use of other turbulence closures was considered, the scale of the problem and the well-established nature of the standard $k-\varepsilon$ closure model made it a suitable choice for this study. Furthermore, Zuckerman and Lior [16] showed that while there exists a tendency for all turbulence models to over-predict the heat transfer in stagnation region, the standard $k-\varepsilon$ model is at the lower end of this spectrum with errors in predicted Nusselt numbers of less than 22\%. It is also noted in Singh et al. [23] that for small jet-to-cylinder spacings $(y / d \leqslant 4)$ there is little variation between the different turbulence models that were studied. Since the largest jet-to-cylinder spacing used in this study is 2.1, the standard $k-\varepsilon$ turbulence model was deemed appropriate. The study of different turbulence models has been done by numerous other authors and is outside the scope of this study. The transport equations for the standard $k-\varepsilon$ closure used herein are [26]:

Turbulent Kinetic Energy:

$\frac{\partial}{\partial t}(\rho k)+\frac{\partial}{\partial x_{i}}\left(\rho k u_{i}\right)=\frac{\partial}{\partial x_{j}}\left[\left(\mu+\frac{\mu_{t}}{\sigma_{k}}\right) \frac{\partial k}{\partial x_{j}}\right]+G_{k}+G_{b}-\rho \varepsilon-Y_{M}+S_{k}$

Dissipation rate:

$$
\begin{aligned}
\frac{\partial}{\partial t}(\rho \varepsilon)+\frac{\partial}{\partial x_{i}}\left(\rho \varepsilon u_{i}\right)= & \frac{\partial}{\partial x_{j}}\left[\left(\mu+\frac{\mu_{t}}{\sigma_{\varepsilon}}\right) \frac{\partial \varepsilon}{\partial x_{j}}\right]+C_{1 \varepsilon} \frac{\varepsilon}{k}\left(G_{k}+C_{3 \varepsilon} G_{b}\right) \\
& -C_{2 \varepsilon} \rho \frac{\varepsilon^{2}}{k}+S_{\varepsilon}
\end{aligned}
$$

where the turbulent eddy viscosity is computed as:

$\mu_{t}=\rho C_{\mu} \frac{k^{2}}{\varepsilon}$

turbulence production is:

$G_{k}=\mu_{t} S^{2}$

and the body force term is:

$G_{b}=\beta g_{i} \frac{\mu_{t}}{P r_{t}} \frac{\partial T}{\partial x_{i}}$

with:

$S \equiv \sqrt{2 S_{i j} S_{i j}}$

$\beta=-\frac{1}{\rho}\left(\frac{\partial \rho}{\partial T}\right)_{p}$

The body force term $G_{b}$ is turned off by default in the turbulence dissipation rate equation. Due to the high flow from the jets, inclusion of this term when solving for turbulence dissipation rate did not influence the results.

\section{Computational model}

The second-order upwind differencing scheme available in ANSYS FLUENT 13.0 was used to model advection in all transport equations. Standard wall-functions were used to bridge the sharp hydrodynamic and thermal boundary layers at all solid surfaces. In this manner, the grid was developed such that $y^{+} \geqslant 30$ for as much as possible of the cylinder surface. The simulation time averaged between 15 and $20 \mathrm{~h}$ per case using a 4-core Intel Core i7-
$38203.60 \mathrm{GHz}$ CPU with 16 GB of RAM running Windows 7 Professional. Multiple cases were also run on the Shared Hierarchical Academic Research Computing Network (SHARCNET) when the grid was larger than $2,000,000$ cells. The working fluid was air and properties were evaluated at a reference temperature of 298.15 K. Thermophysical properties for air are given in Table 1.

The model geometry shown in Fig. 4 was designed and modified using SolidWorks 2013 where it was then exported as a PARASOLID $^{\mathrm{TM}}$ file and imported into ANSYS ICEM ${ }^{\mathrm{TM}}$ for mesh generation. Fig. 5 shows the geometry and surface meshes on the segment shown in Fig. 4. The computational domain was discretized using tetrahedral cells wherein a fine mesh size was used at the cylinder surface and in the vicinity of the jet exit to accommodate the sharp velocity and temperature gradients that were expected in the impingement and entrainment regions. A grid utilizing prism elements on the cylinder surface transitioning to tetrahedral elements was also tested for one configuration. As the prism mesh produced changes of less than $0.5 \%$ in heat transfer, only pure tetrahedral meshes were used. Even with the pure tetrahedral meshes, there was no difficulty in maintaining a fine grid spacing at the cylinder wall to be in the proper range of $y+$. A grid independence study, described in detail below, was performed to ensure accuracy of the computational results to within $1 \%$ based on overall heat flux from the cylinder.

Boundary conditions were imposed on all external faces of the computational domain, as shown in Fig. 6. Flow at the jet inlet was set using a velocity inlet condition, with a uniform velocity.

Table 1

Thermophysical properties of air at $298.15 \mathrm{~K}$.

\begin{tabular}{ll}
\hline Property & Value \\
\hline Density $\left(\mathrm{kg} / \mathrm{m}^{3}\right)$ & 1.225 \\
Specific heat $(\mathrm{J} / \mathrm{kg} \mathrm{K})$ & 1006.43 \\
Thermal conductivity $(\mathrm{W} / \mathrm{m} \mathrm{K})$ & 0.0242 \\
Kinematic viscosity $(\mathrm{kg} / \mathrm{m} \mathrm{s})$ & $1.7894 \mathrm{e}-05$ \\
\hline
\end{tabular}
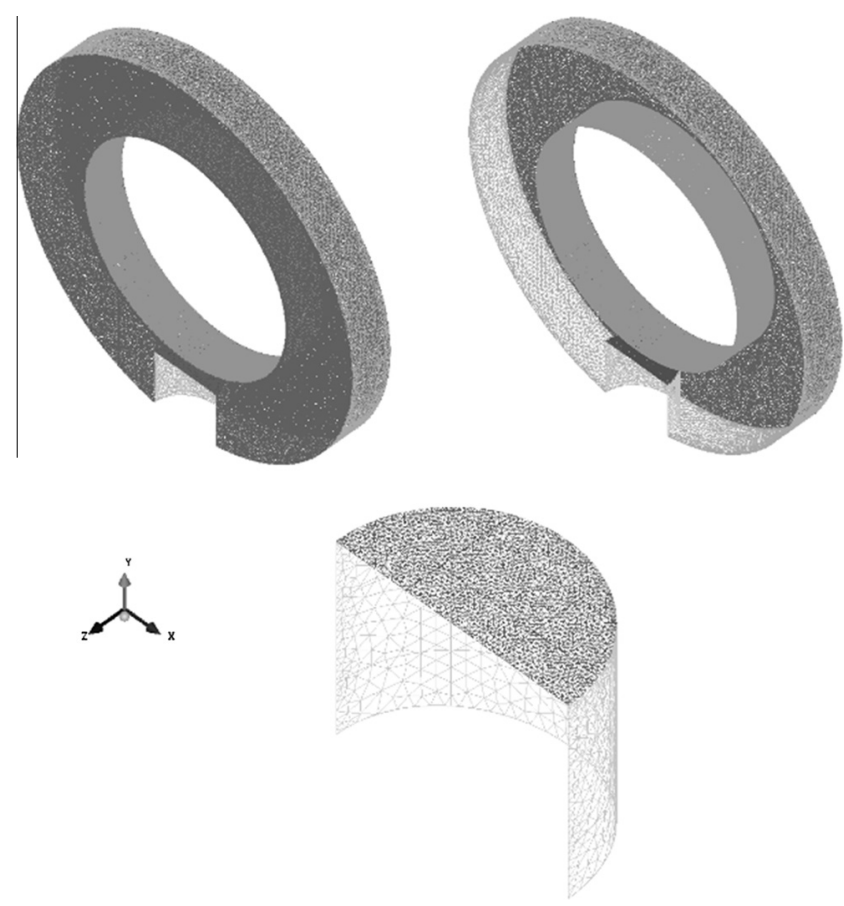

Fig. 5. Views of surface meshes generated for reduced computational domain; the lower image is a close-up of the inlet jet region. 


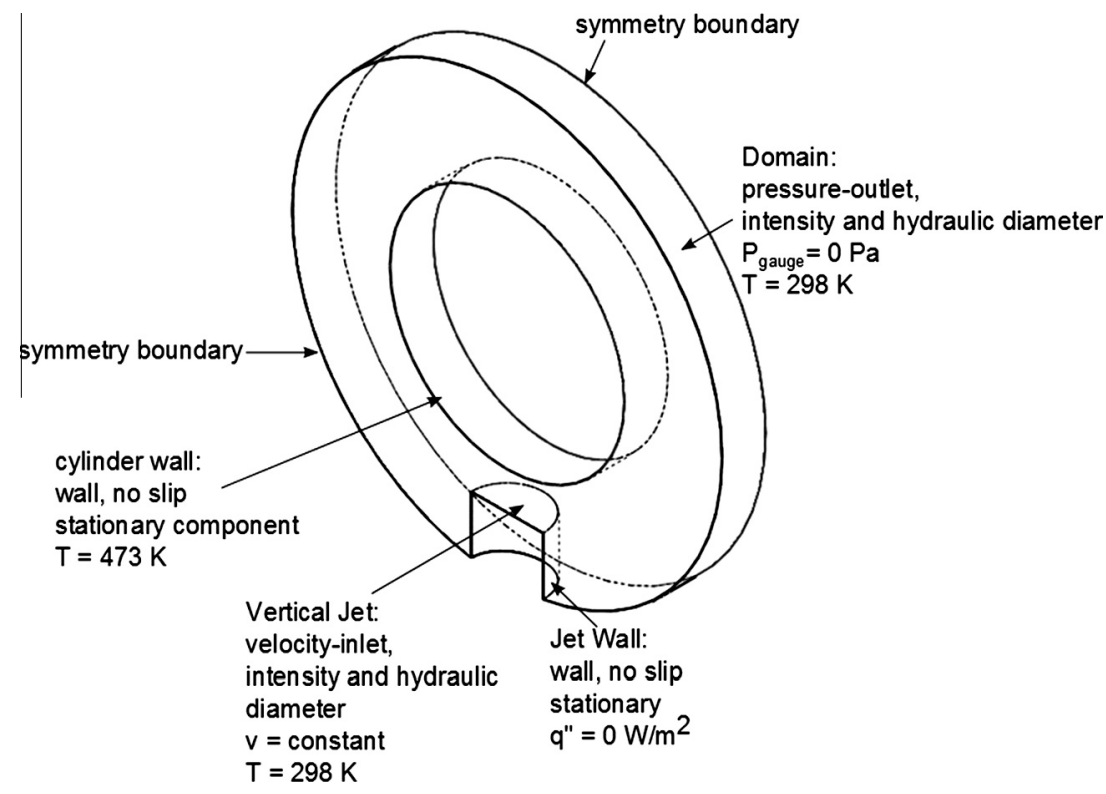

Fig. 6. Boundary condition description for CFD simulations.

The jet velocity condition was based on an area weighted average of measured outlet velocities from jets utilized at a cement production facility. The fans used at the facility were comprised of long $(8 \mathrm{~m})$ cylindrical ducts with fans drawing in air at their base. The air passed across the electric fan motor and through straighteners before exiting at the outlet, which is the location where the vertical jet velocity is imposed, as seen in Fig. 6. Measurements across the outlet of the fan revealed a flat parabolic profile, which we felt would be most generally implemented in the computational model as a uniform plug flow. This simplification facilitates the consideration of different sized jets without scaling, and makes the computational study easily reproducible. While a study of the effect of jet profile would also be useful, it is outside the scope of the current study. Velocity values of order $10 \mathrm{~m} / \mathrm{s}$ were measured; this value was also modified to accommodate a suitable range of Reynolds numbers. The turbulence intensity at the velocity inlet was set to a high value to accommodate the large diameter of the jets and distance from the fan to the jet outlet; assignment of the proper value is considered below in the model verification. The cylinder wall was set as a stationary, no-slip, impermeable wall with a constant temperature of $473 \mathrm{~K}$ for all cases; the temperature is based on the target average shell temperature for a typical cement kiln. In terms of the stationary assumption, we refer back to the work of Jeng et al. [10], who reported that the effect of rotational Reynolds number on convective heat transfer decreases as jet Reynolds number increases. Since the present cases consider a high jet Reynolds number, and the rotational speed of the cylinder is small in practice, the effect of rotation on heat transfer should be negligible. As a test, simulations were performed for one case at rotational speeds of 1.5 and $3 \mathrm{rpm}$, which is a typical rotational speed for a cement kiln. Heat flux results were predicted to within $1 \%$ of the results of a stationary cylinder, corroborating the assumption that the effect of rotation on heat transfer is negligible for the cases considered herein. The outer circular surface of the domain was modeled as a constant-static-pressure boundary to account for outflow and backflow. The pressure for this boundary was set to ambient static pressure [16] with a backflow temperature of $298 \mathrm{~K}$, and backflow turbulence intensity of 5\%. Symmetry conditions were imposed on the two axial planes.

A grid-independence study was performed to determine the grid density and turbulence intensity required to produce overall heat transfer rates to within 5\%. This study also considered inlet turbulence intensity values of $5 \%$ and $10 \%$ on each grid studied. The geometry considered is a vertical jet centered under the cylinder axis with $y / d=0.52, z / d=1.2$, and $R_{d}=1,236,000$. Fig. 7 shows the variation of local Nusselt number along the $z$-axis of the cylinder for different grid sizes and inlet turbulent intensities of $5 \%$ and $10 \%$. Considering first the series of computations done for a turbulence intensity of $10 \%$, Fig. 7 shows that with each subsequent grid refinement, the profile of axial local Nusselt number, $\mathrm{Nu}_{z}$, becomes more and more smooth, i.e. the large variations of $N u_{z}$ with $z / D$ become progressively smaller with increasing grid density. Differences of approximately $5 \%$ are observed between the $1,750,000$ and 6,500,000 element grids. The computations done on the two finest grids for $5 \%$ and $10 \%$ turbulence intensity show additional small changes in the local Nusselt number, but no change in the distribution with respect to $z / D$. The local differences shown in Fig. 7 between the 1,750,000 and 6,500,000 element grids and the $5 \%$ and $10 \%$ turbulence intensities result in less than a $1 \%$ difference in the overall Nusselt number. As the primary focus of this study is the overall Nusselt number, a grid-density based on $1,750,000$ elements with a turbulence intensity of $10 \%$ was used for all subsequent calculations. Since the domain size varied with the $z / d$ parameter, the chosen grid-density resulted in overall grid sizes between 945,000 elements and 4,660,000 elements. The position of the outer boundary of the domain was also studied separately to ensure that its position did not influence the heat

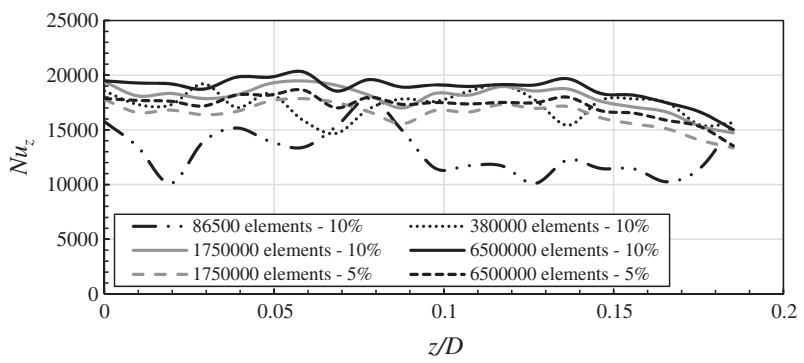

Fig. 7. Plot of local Nusselt number as a function of axial distance. Plot shows variation of turbulent intensity and different grid sizes in grid independence model. 
transfer results at the cylinder. At the highest Reynolds number considered, outer domain diameters beyond $2.2 \mathrm{D}$ produced insignificant changes in the local and overall heat transfer results.

\subsection{Validation of numerical model}

As detailed experimental results are not available for the exact cases considered, the numerical model is verified by comparing values of stagnation Nusselt number against the work of Singh et al. [23] for a single centered jet with a $y / d$ ratio of 4 and $d / D$ value between 0.11 and 0.2 . The jet Reynolds number was matched at values of $10,000-25,000$, and the turbulence intensity was fixed at $10 \%$, as noted above. Fig. 8 shows present numerical results for $d / D=0.153$, and experimental and numerical results from Singh et al. [23] for $d / D=0.11$ and 0.2 . The figure indicates that the general trend of increasing $N u_{\text {stag }}$ with increasing $R e_{d}$ is correctly predicted. The figure also shows that while the experimental results of Singh et al. [23] are considerably lower than their own numerical results for both $d / D$ cases considered, the current numerical results for $d / D=0.153$ across the full range of $R e_{d}$ fall between the numerical results for $d / D=0.11$ and $d / D=0.2$, as they should. The overprediction of heat transfer is mainly due to the over-prediction of turbulence in the stagnation region [16].

A further validation case was run to consider two jets with a large axial spacing so that the results could be compared to the single impinging jet results of Singh et al. [23]. A spacing of $z / d=7$ was used to ensure that the regions of impingement were not impacted by the neighboring jets. The results in Fig. 9 are in terms of $z / D$ and are only given to $z / D=0.45$. Beyond this value, the air stream from neighboring jet influences the jet in question and is, thus not relevant in comparison with the single jet case of Singh et al. [23]. The results in Fig. 9 show that the values of stagnation Nusselt number for the numerical model used are within the same range as the numerical results of Singh et al. [23]. Fig. 9 also shows that beyond $z / D=0.2$ the axial variations in Nusselt number match the results of Singh et al. [23] extremely well; this is also the region where the experimental results best match the numerical results. It can be concluded from the validation cases that the present numerical model, the domain and boundary conditions, and the computational mesh produce results that are accurate in comparison to previous numerical and experimental work and are thus sufficient for the proposed study.

Though the computational model has been validated against existing work, it is important to note here that since direct validation is not possible for the specific cases studied herein, the CFD

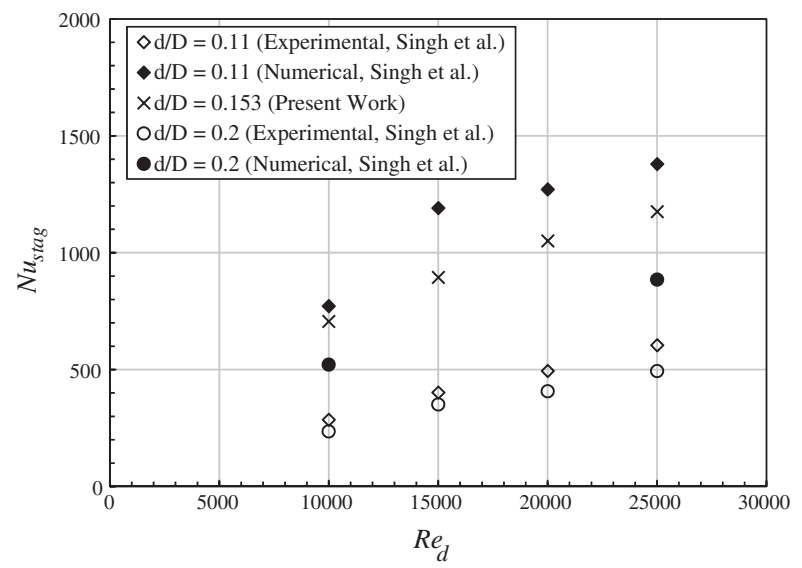

Fig. 8. Comparison of stagnation $\mathrm{Nu}$ predicted by present numerical model to experimental and numerical results of Singh et al. [23] for a single centered vertical jet at $y / d=4$.
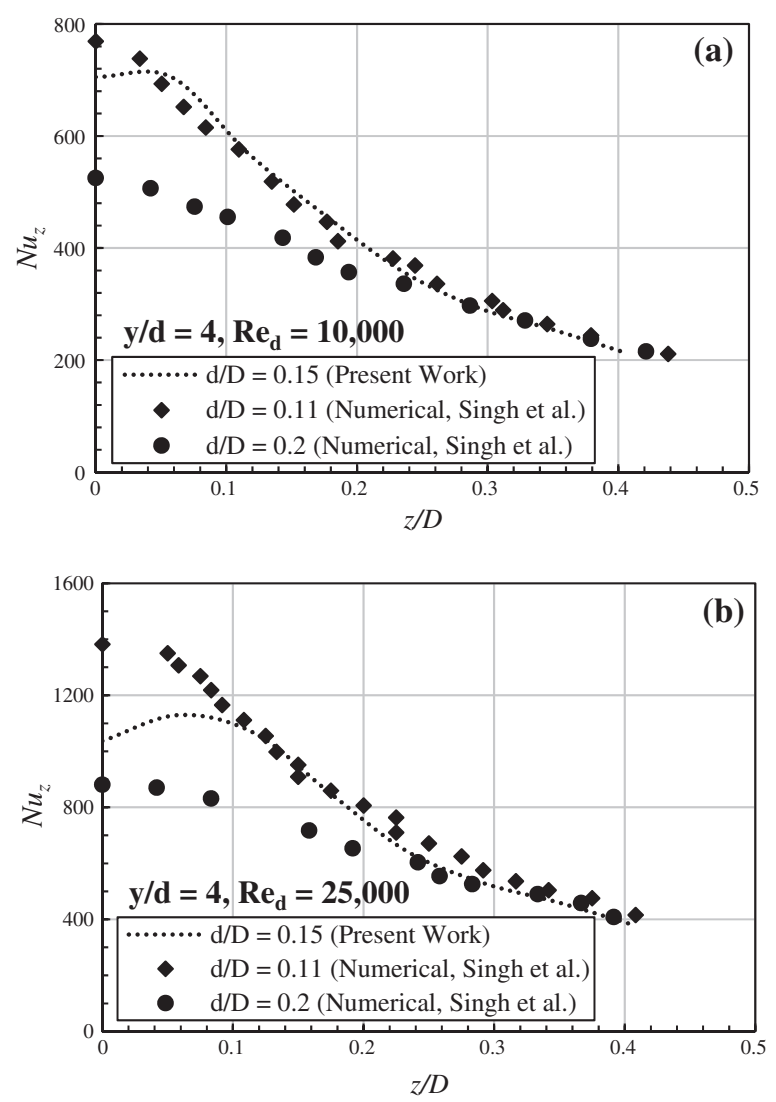

Fig. 9. Comparison of axial Nusselt number for validation case of $y / d=4$ and (a) $R e_{d}=10,000$ and (b) $R e_{d}=25,000$.

results be considered most accurate in terms of predicting differences between cases, as opposed to absolute heat transfer results. To this end, the most important aspect of the computed results are the trends observed when varying the geometric parameters.

\section{Results and discussion}

A parametric study on the effect of forced convective cooling due to multiple jets impinging on a horizontal cylinder has been carried out. The parametric ranges considered are as follows:

- Jet-to-cylinder spacing ranged from $0.2 \leqslant y / d \leqslant 2.1$

- Jet-to-jet spacing ranged from $1.21 \leqslant z / d \leqslant 3.41$

- Ratio of jet diameter to cylinder diameter ranged from $0.15 \leqslant d /$ $D \leqslant 0.31$

- Jet offset from the cylinder axis ranged from $0 \leqslant x$ | $d \leqslant(D-d) / 2 d$, given by Eq. (1).

- Reynolds number range $2.06 \times 10^{5} \leqslant R e_{d} \leqslant 1.236 \times 10^{6}$, where $R e_{d}$ is based on the jet diameter $d$, the uniform jet exit velocity $V_{j}$, and the kinematic viscosity of air (see Table 1 ):

$R e_{d}=\frac{V_{J} d}{v}$

For each case computed, the overall (average) heat transfer coefficient, and the overall Nusselt number were calculated according to:

$h_{a v e}=\frac{q^{\prime \prime}}{\left(T_{c y l}-T_{j}\right)}$

$N u_{a v e}=h_{a v e}\left(\frac{D}{k_{f}}\right)$ 
Table 2

Jet Reynolds numbers at various air stream velocities.

\begin{tabular}{rrrl}
\hline Jet velocity, $V_{j}(\mathrm{~m} / \mathrm{s})$ & \multicolumn{3}{c}{ Jet Reynolds number, $R e_{d}$} \\
\cline { 2 - 4 } & $d / D=0.31$ & $d / D=0.23$ & $d / D=0.15$ \\
\hline 5 & 412,000 & 309,000 & 206,000 \\
10 & 823,000 & 617,250 & 411,500 \\
15 & $1,236,000$ & 927,000 & 618,000 \\
\hline
\end{tabular}

As the velocity of the jets in operation was measured to be approximately $10 \mathrm{~m} / \mathrm{s}$, the range of jet velocity was chosen to be $5 \leqslant V_{j} \leqslant 15 \mathrm{~m} / \mathrm{s}$. Table 2 shows the $d / D$ ratios considered and the corresponding Reynolds numbers (recall that the cylinder diameter is fixed at $D=4.8 \mathrm{~m}$ ). The results are presented in a systematic manner by describing the influence of the different geometric parameters.

\subsection{Effect of jet-to-cylinder spacing, $y / d$}

Fig. 10 shows the effect of varying $y / d$ over the ranges of $x / d, z / d$ and $R e_{d}$ considered. Each pair of plots shows the heat transfer trends for $x / d=0$ (left) and $(x / d)_{\max }$ (right). As mentioned previously, $(x / d)_{\max }$ corresponds to the case when the edge of the vertical jet is adjacent to the outer edge of the cylinder. In Fig. 10(a)-(d), it can be seen that for a row of jets of dimension $d / D \geqslant 0.23$, the overall Nusselt number $\left(N u_{\text {ave }}\right)$ decreases with increasing $y / d$ for all $z / d$ considered, and that the reduction in $N u_{a v e}$ decreases with decreasing jet Reynolds number. This is the case for both the centered $(x / d=0)$ and fully offset conditions. Gardon and Akfirat [7] also observed a decrease in heat transfer with increasing impingement distance for small jet-to-surface distances in the potential core region of a slot-jet impinging on a flat plate, whereas, Singh et al. [23] reported similar behavior for a circular jet impinging on a cylinder. Singh et al. [23] described this decrease as resulting from an increasing jet spreading with increasing jet-to-cylinder separation distance. It is also observed that as $d / D$ decreases from 0.31 to 0.23 , the reduction in $N u_{\text {ave }}$ due to increasing $y / d$ decreases; in fact, at an axial separation of $z / d=3.4$, the difference in $N u_{\text {ave }}$ between $y / d=0.3$ and 1.4 is almost negligible at $(\mathrm{x} / \mathrm{d}) \max$. Fig. 10 (e) and (f) shows that when $d / D=0.15$, there is a region between $1.6<z / d<1.8$ where $N u_{\text {ave }}$ actually increases with increasing $y / d$. Here, an enhancement in $N u_{\text {ave }}$ (between $y / d=0.4$ and 2.1) occurs for all Reynolds numbers at approximately $z / d=1.6$. This trend is observed for both the centered and fully-offset cases in Fig. 10 (e) and (f), except that the enhancement at $z / d=1.6$ is most significant for $x / d=0$. The trends shown in Fig. 10 suggest that as $y / d$ increases, there are cases where a local maximum in $N u_{\text {ave }}$ is achieved, however, it is also apparent that this local maximum is dependent upon the $z / d$ and $d / D$ ratios, and location of the jet with respect to the centerline of the cylinder.

To reconcile the heat transfer behavior and the local maximum in $N u_{\text {ave }}$, it is necessary to consider the proximity of the jet outlet with the cylinder and the related flow structure in the
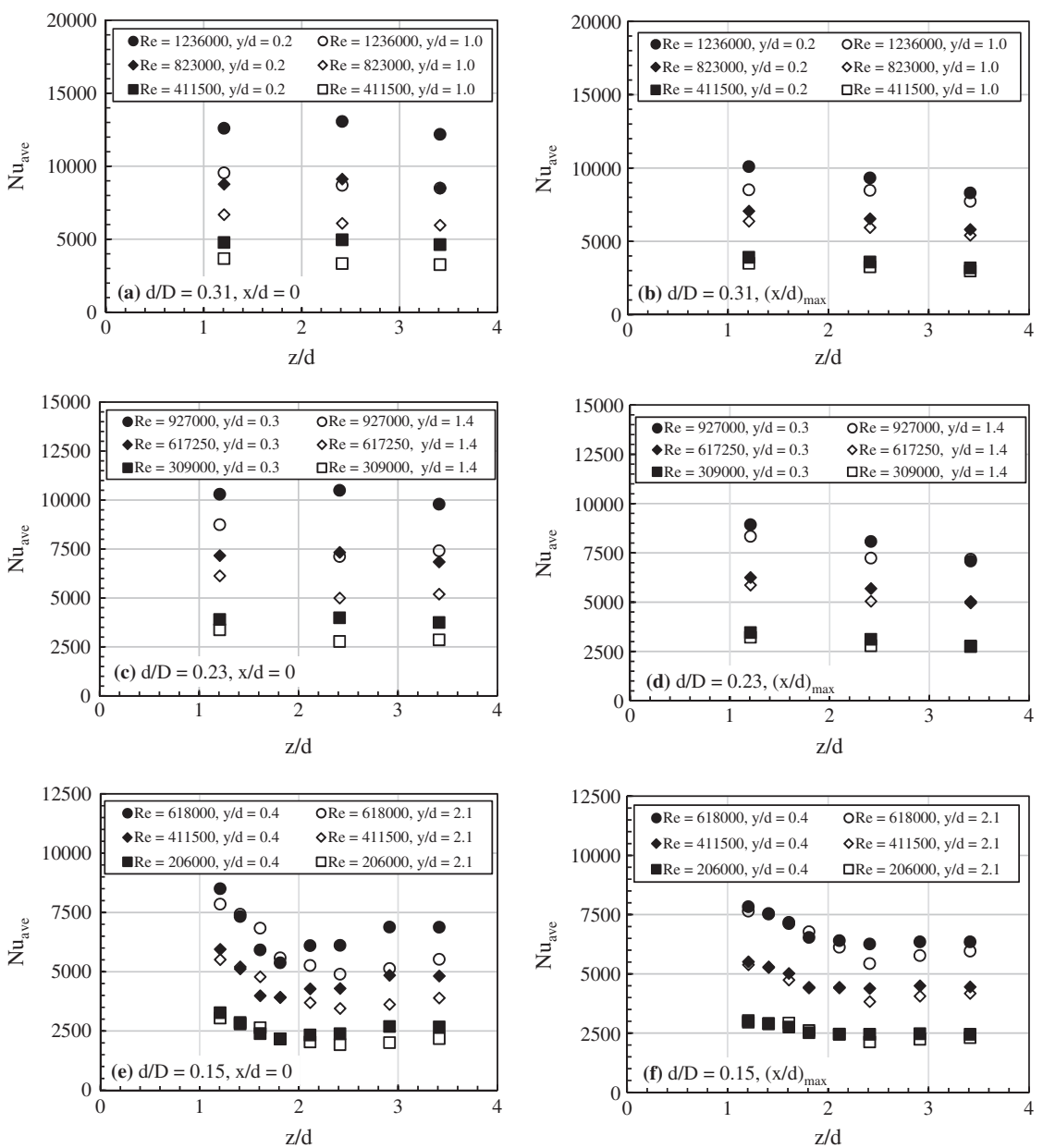

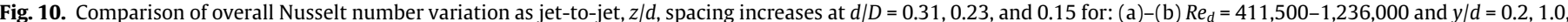

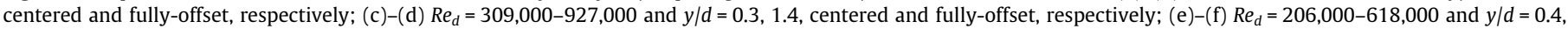
2.1 , centered and fully-offset, respectively. 
(a)

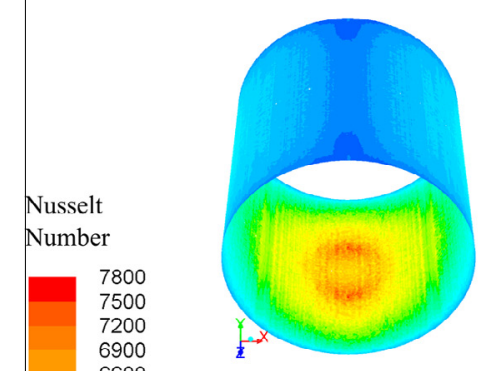

(c)

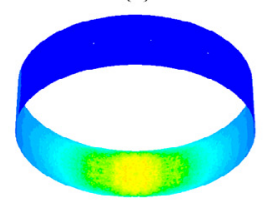

$\sum_{2}^{2}$

(e)

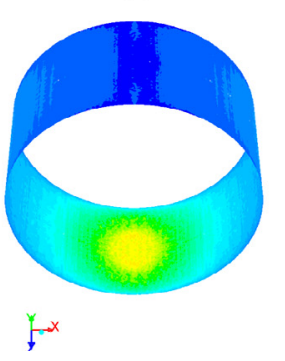

(b)

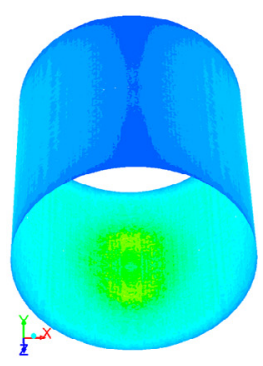

(d)

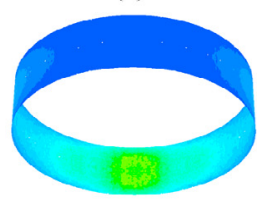

(f)

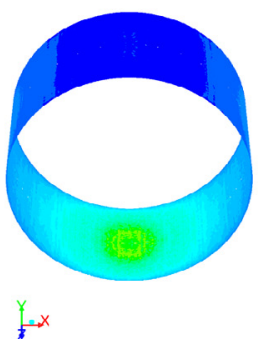

Fig. 11. Images (a)-(b) show contours of Nusselt number for $R e_{d}=1,236,000, d$ / $D=0.31, z / d=3.4, x / d=0$, and $y / d=0.2$ and 1.0 , respectively. Images (c) $-(\mathrm{d})$ show streamlines for $R e_{d}=618,000, d / D=0.15, z / d=1.6, x / d=0$, and $y / d=0.4$ and 2.1, respectively. Images (e)-(f) show streamlines for $R e_{d}=618,000, d / D=0.15, z / d=3.4$, $x / d=0$, and $y / d=0.4$ and 2.1 , respectively.

impingement region and around the cylinder. For a single circular jet, potential core lengths have been measured to be $y / d \approx 4[3,21]$. Thus, for the close proximity of the jet with the cylinder considered herein $(0.2 \leqslant y / d \leqslant 2.1)$, the impingement region occurs within the potential core for all parameters studied. This is a unique aspect of the present study compared to all previous work where the jet is typically positioned such that the impingement region transitions from the tip of the potential core to well outside of it [16-23]. Increasing $y / d$ within the potential core has a significant effect on the flow structure and the heat transfer from the cylinder. Nada [15] observed that as the tip of the potential core is approached, the effect of the turbulent shear layer on the cylinder is greater resulting in higher convective heat transfer.

Fig. 11 shows surface heat transfer contours for a jet size of $d /$ $D=0.31$ ( $\mathrm{a}$ and $\mathrm{b}$ ) and $d / D=0.15$ (c and f). For the case shown in ( $a$ and $b$ ), the large jet is positioned under the center axis of the cylinder $(x / d=0)$, where $R e_{d}=1,236,000, z / d=3.4$, and $y / d=0.2$ and 1.0. These parameters lie within the region described in Fig. 10(a)-(d) where increases in any parameter led to a decrease in overall heat transfer. In these cases, the cylinder lies well inside the shear layer for both $y / d$ positions shown, and the potential core nearly covers the entire impingement region on the upstream side of the cylinder.

Fig. 12(a) and (b) shows sectional streamline plots for the same cases shown in Fig. 11(a) and (b). Here it is seen that for both $y / d$ shown, a strong and energetic turbulent flow remains attached to the cylinder, producing only a small wake on the leeward side.

Fig. 13 shows the pressure coefficients for the cylinder in Fig. 11 (f). Referring back to the Coanda effect, and the work of Kumada et al. [19], Fig. 13 shows large pressure gradients in the stagnation region. A negative pressure coefficient is observed at the region where flow remains attached, as seen in Fig. 12(f), while a positive pressure coefficient is observed after the flow separates from the cylinder. Due to the proximity of the nearby jets, a secondary positive pressure coefficient is observed in a secondary stagnation region where the axial airflow from each jet impinges upon one another.

The case of $d / D=0.15$ shown in Fig. 11(c)-(f) yields a different result for heat transfer and flow. Images (c)-(d) show heat transfer contours for $\operatorname{Re}_{d}=618,000, z / d=1.6$ and $y / d$ of 0.4 and 2.1, while images (e)-(f) show the same jet condition, except with $z / d=3.4$. Comparison of (c) and (d) shows that while the impingement region has higher heat transfer for $y / d=0.4$, the heat transfer is better distributed for $y / d=2.1$, thereby leading to a higher average heat transfer, as indicated in Fig. 10(e). Fig. 12(c) and (d) shows sectional streamlines for these cases and clearly indicates a small wake on the leeward side for the large vertical spacing, (d), and a much wider wake for the near spacing (c). The streamline plot indicates that for the near case shown in Figs. 11 and 12(c), the flow is deflected away from the cylinder producing a large wake that is filled with air moving axially, as suggested by the streamlines seemingly originating at the cylinder wall. This results in low heat transfer over a good portion of the cylinder and lower average heat transfer than for $y / d=2.1$.

The images in Fig. 11(e) and (f) show that for a higher axial spacing, the heat transfer around the cylinder is fairly well distributed for both cases. Not surprisingly, Fig. 12(e) and (f) shows that the jet and entrainment flow for these cases produce similarly narrow wakes on the leeward side, although the larger spacing case has a slightly larger wake. Recall that for this axial spacing, the heat transfer decreased with increasing vertical distance, $y / d$, similar to that observed for the larger jets. In this respect, the axial jet spacing appears to have an important influence on the flow structure and the resulting heat transfer.

\subsection{Effect of jet offset, $x / d$}

For each $d / D$ ratio, the effect that offsetting the jets had on heat transfer was studied for various jet-to-cylinder, $y / d$, and axial, $z / d$, spacings. Fig. 14 shows the data from Fig. 10, except plotted to show the influence of increasing $x / d$ at a constant $y / d$ and $z / d$. Fig. $14(a)-(d)$ shows that for the larger jets $(d / D=0.31,0.23)$, the heat transfer decreases with increasing $x / d$ for all conditions shown. For $d / D=0.15$, however, the heat transfer increases with jet offset for certain other parameters. Fig. 14(e) shows that at $y$ / $d=0.4$ the heat transfer increases with jet offset in the range $1.4<z / d<2.4$, beyond which the trend reverses. Fig. 14(f) shows that for $y / d=2.1$, the fully-offset case has a higher heat transfer for the full range of $z / d$ considered. Fig. 15 shows a comparison of a large jet case with $d / D=0.31$ at $R e_{d}=1,236,000$ with the small jet case of $d / D=0.15$ at $R_{d}=618,000$, both for an axial spacing of $z /$ $d=3.4$. Though the $R e_{d}$ is different for the two cases, the velocity of the airflow at the jet exit is the same for both. In addition, both cases are fully-offset and $y / d=1.0$ for the large jet (a) and $y /$ $d=2.1$ for the small jet (b), to maintain the same vertical spacing for both cases. The figure shows the difference in impingement and wake structures for the two cases. In plot (a), it is clearly seen that because of the large diameter ratio of the jet, the impingement still results in part of the jet flowing towards the cylinder axis, thereby producing a region of attached flow on the left side of the jet combined with a strong energetic region on the right side. It can be imagined from this image that for the given $x / d$, a decrease in $y / d$ would reduce the flow towards the axis of the 
(a)

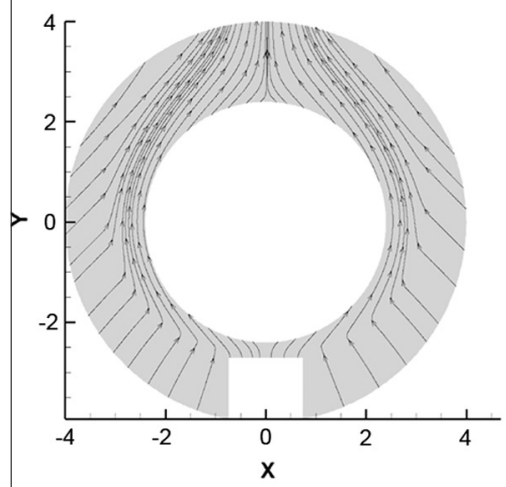

(c)

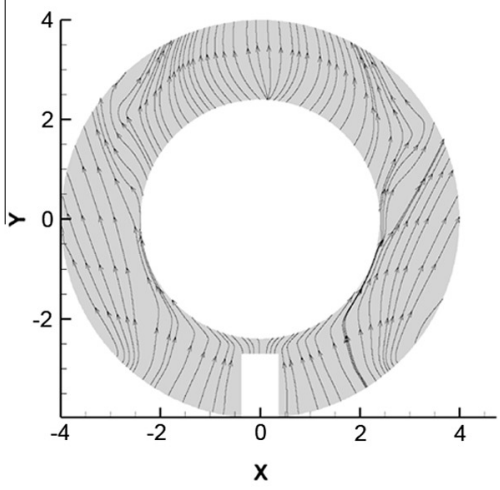

(e)

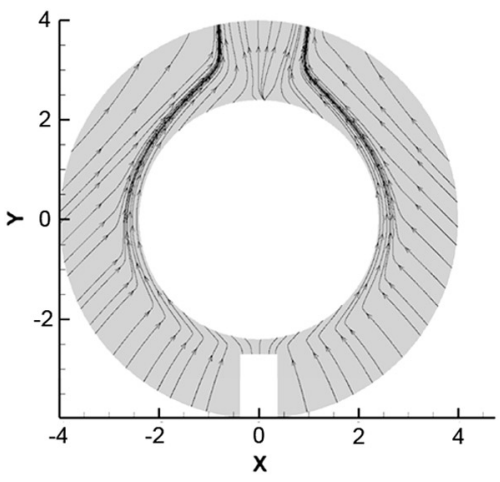

(b)

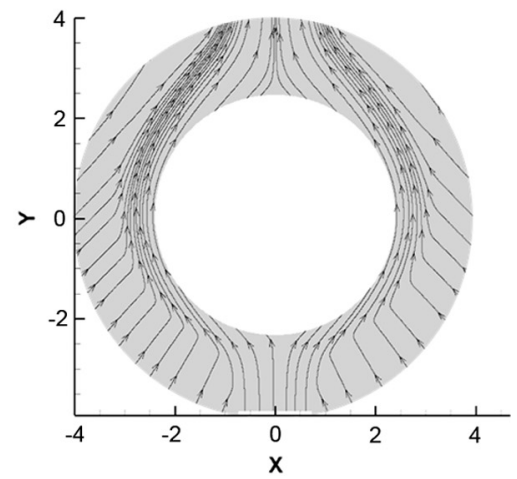

(d)

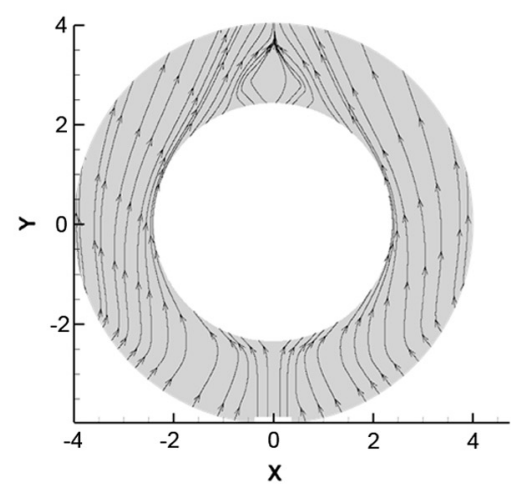

(f)

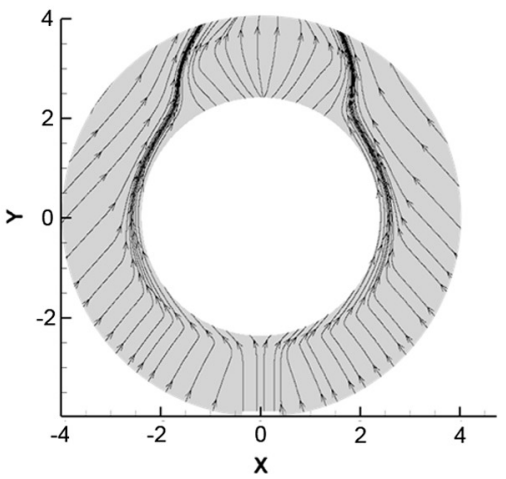

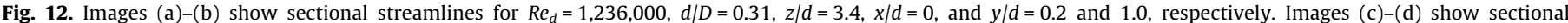

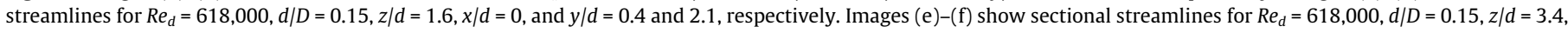
$x / d=0$, and $y / d=0.4$ and 2.1 , respectively.

cylinder, while an increase would increase this flow, due to the width of the jet at the impingement region. The result is a significant wake on the leeward side of the cylinder - in comparison to that shown in Fig. 12(a) - and a reduction in heat transfer compared to the case of the axially centered jet (Figs. 11a and 12a). This trend is similar for all the cases of $d / D \geqslant 0.23$ for the entire ranges of $x / d, y / d$ and $z / d$ considered; i.e. an increase in any of the geometric parameters for $d / D \geqslant 0.23$ led to a reduction in heat transfer.

The flow structure seen in Fig. 15(b) shows that, in contrast to the large jet case, there is almost no flow from the impingement region directed towards the cylinder axis, and the flow on the left side of the cylinder is mainly axial, as indicated by the sectional streamlines. This flow structure is similar to that described by
Wang et al. [21], where it was seen for the jet spacing $y / d=2.0$, that as jet inclination increased (analogous to $x / d$ offset), flow separation did not occur. As the inclination angle increased, the tangential local Nusselt number increased in the region where the flow remains attached to the cylinder increase; an increase in stagnation Nusselt number was also reported. A similar trend is seen in Fig. 15(b), as the flow on the right side is strong and energetic, resulting in higher heat transfer than the case shown in Fig. 12 (f). Interestingly, this flow field leads to higher heat transfer than the case of $x / d=0$, with all other parameters remaining the same. It is thought that the significant region of high-energy attached flow for the offset cases gives the net effect of a higher average heat transfer than the jets centered axially under the cylinder at the same $y / d$, as indicated in Fig. 14(f). 
Pressure Coefficient
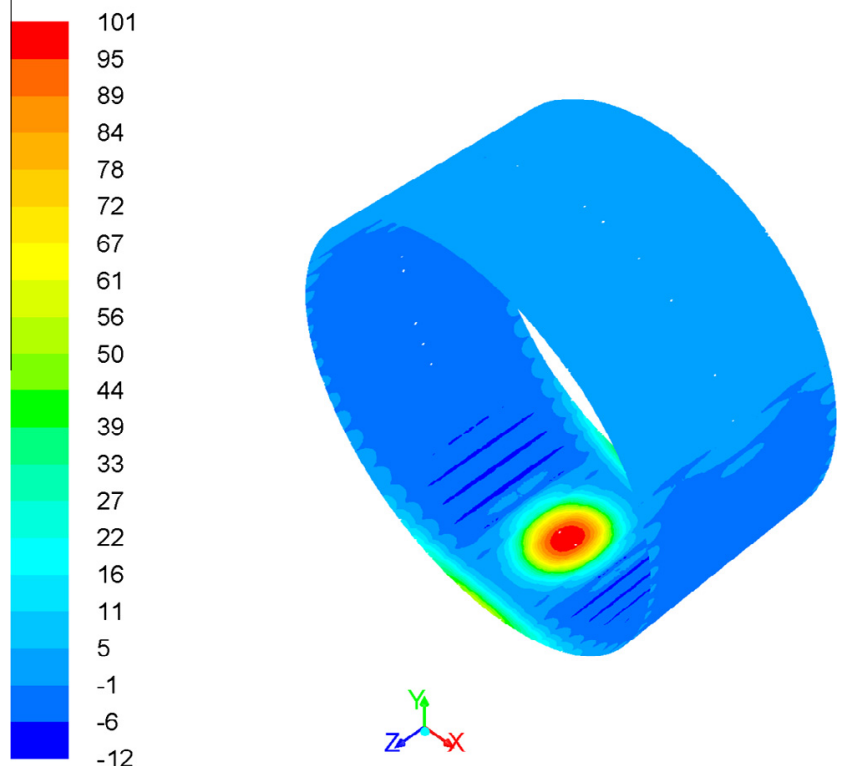

Fig. 13. Isometric view of pressure coefficient values over cylinder when $R e_{d}=618,000, d / D=0.15, z / d=3.4, x / d=0$, and $y / d=2.1$.
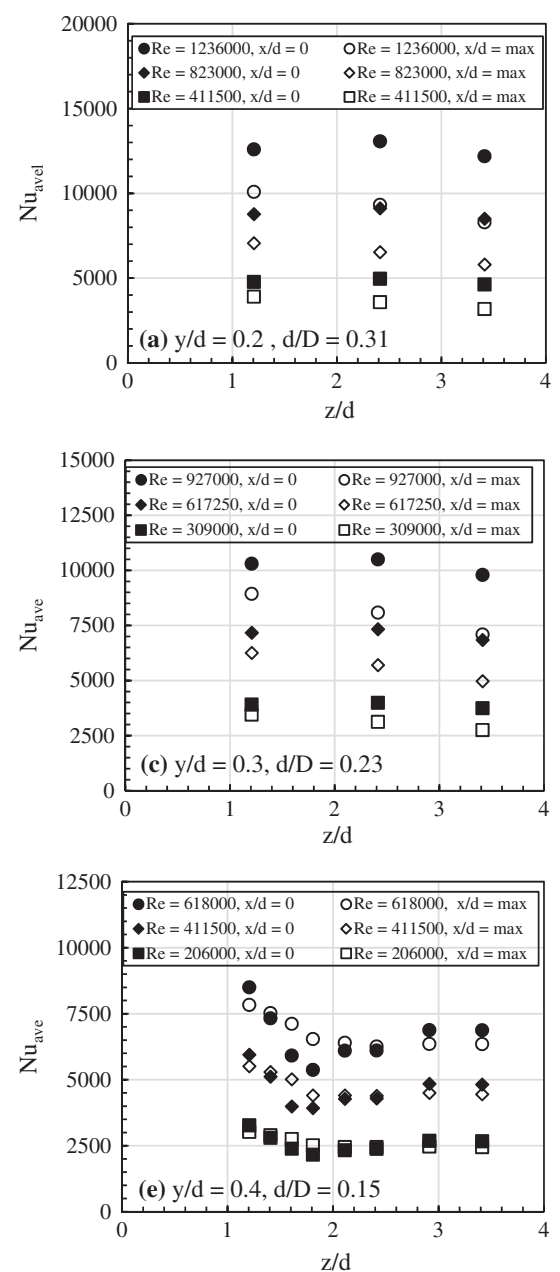

\subsection{Effect of axial jet spacing, $z / d$}

As seen previously, each case was studied at multiple different $z / d$ ratios. It can be seen in Figs. 10 and 14 that the trend for $d /$ $D \geqslant 0.23$ is a decrease in average Nusselt number with respect to increasing $z / d$; and for $d / D=0.15$ the trend varies. The work of Dong et al. [24] displays a similar trend for multiple circular jets impinging on a flat plate. In this work three different axial separations, $z / d$, were studied. What he observed is that from $2.6 \leqslant z /$ $d \leqslant 5$ there is an increase in the area-averaged heat flux, while from $5<z / d \leqslant 7$ there is a decrease. Furthermore, according to the work of Wang et al. [20], circular jets impinging on a cylinder where $d / D \leqslant 0.5$ follow heat transfer characteristics that are apparent when a circular jet impinges on a flat plate. Based on this finding, it is not surprising that for the $d / D$ ratios studied herein, there are combinations of parameters that lead to both increasing and decreasing trends in heat transfer.

Fig. 16 shows sectional streamline plots for three different axial separations for the small jet $(d / D=0.15)$, at $R e_{d}=618,000$ with $y /$ $d=0.4$ and $x / d=0$. The plots give sectional streamlines on the axial center plane to show the interaction between neighboring jets with respect to axial separation. When the jets are closest together, there is no downward flow between them along the center axis, implying that flow is entrained from below and laterally and moves in a weak pattern around the cylinder having little
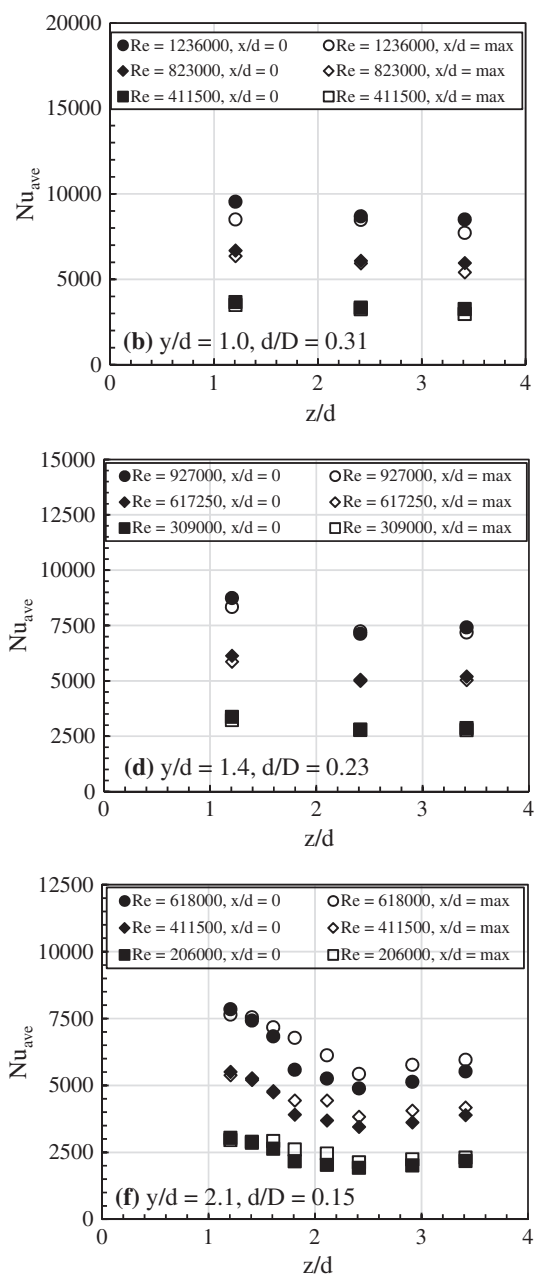

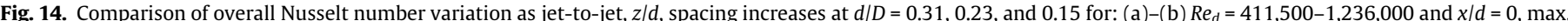

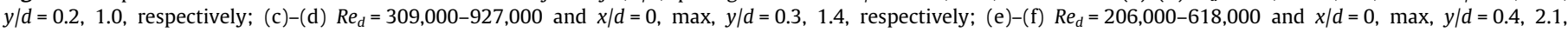
respectively. 


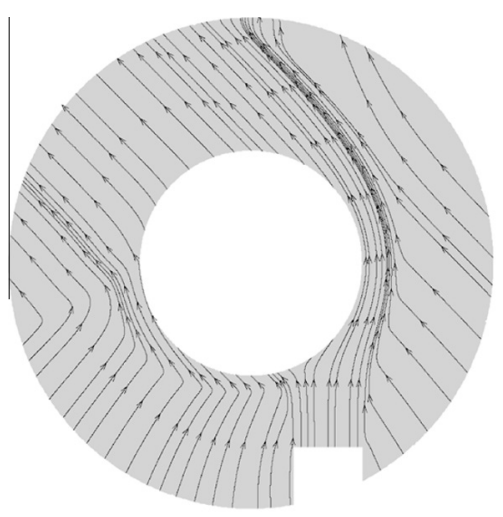

(a)

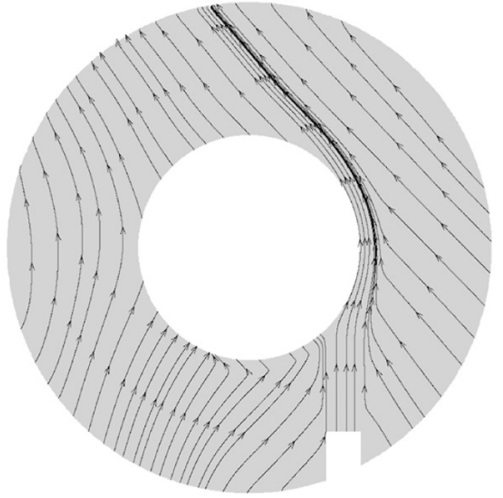

(b)

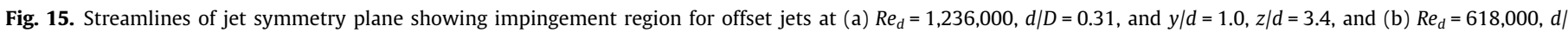
$D=0.15, y / d=2.1, z / d=3.4$

(a)

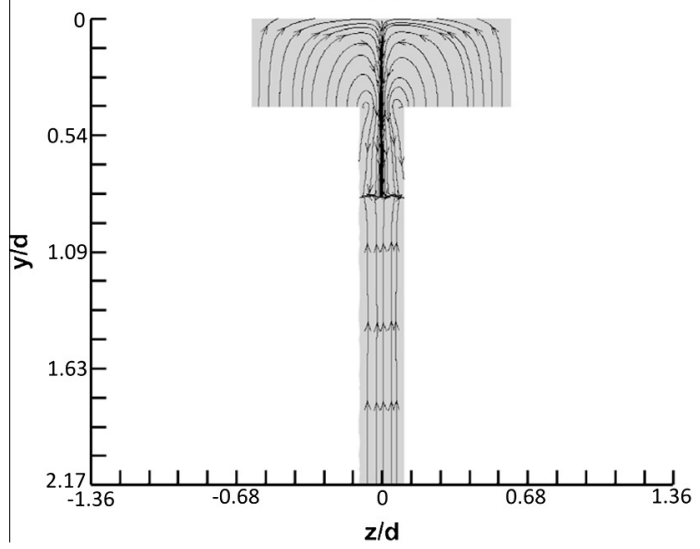

(b)

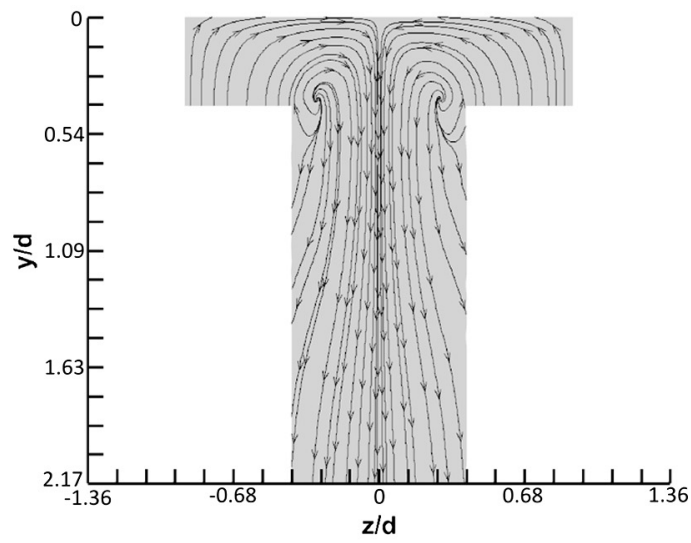

(c)

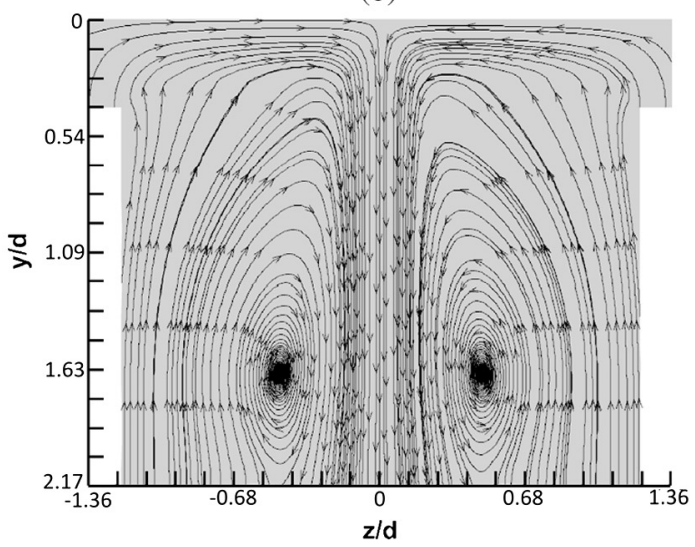

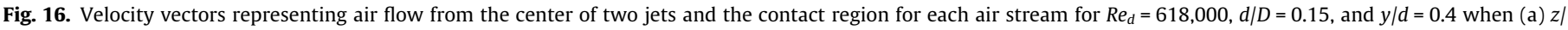
$d=1.2$, (b) $z / d=1.8$, and (c) $z / d=3.4$.

attachment and a large leeward wake, as seen in Fig. 12(c). At the middle axial spacing $(z / d=1.8)$, the jets interact causing an organized downward flow between them; this condition fosters a better attachment to the cylinder, a smaller wake, and higher average heat transfer with increasing $y / d$. At the largest axial spacing considered $(z / d=3.4)$, the jets interact forming a downward flow, but because of the large spacing between them, a large secondary vortex appears which is driven by the downward flow and the weak upward entrained flow along the jet wall. For the centered condition, this condition always leads to a decrease in the average heat transfer.

\subsection{Correlation for average Nusselt number}

The results of average heat transfer for the entire parametric range considered can be concisely described by a correlation that puts the average Nusselt number as a function of Reynolds number, $R e_{d}$, jet-to-cylinder ratio $d / D$, vertical spacing $y / d$, lateral offset 


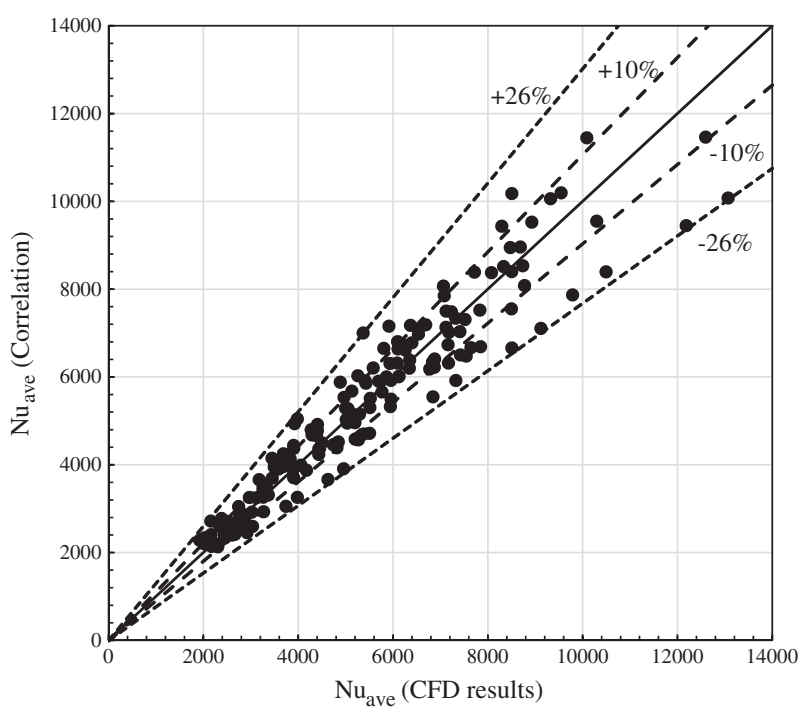

Fig. 17. Correlation for overall Nusselt number based on numerical simulations.

$x / d$, and axial spacing $z / d$. The correlation was produced using the regression analysis package available in Microsoft Excel ${ }^{\mathrm{TM}}$ and is expressed in power-law form as:

$$
\begin{aligned}
N u_{\text {ave }}= & 0.041 R e^{0.862}\left(\frac{d}{D}\right)^{-0.317}\left(\frac{y}{d}\right)^{-0.0729}\left(\frac{z}{d}\right)^{-0.186} \\
& \times \exp \left[-0.00134\left(\frac{x}{d}\right)\right]
\end{aligned}
$$

where, the range of applicability is:

$0.15 \leqslant d / D \leqslant 0.31 ; 0.2 \leqslant y / d \leqslant 2.1 ; 1.21 \leqslant z / d \leqslant 3.41 ; 0 \leqslant x / d \leqslant$ $(D-d) / 2 d$, and for the Reynolds number range: $2.06 \times 105 \leqslant$ $R e_{d} \leqslant 1.236 \times 106$.

Note that the lateral offset $(x / d)$ is included in an exponential function because of the fact that it should have no influence on $N u_{\text {ave }}$ at $x=0$. Fig. 17 shows the results predicted by the correlation mapped against the complete set of computational results. The plot shows that the heat transfer expression given in Eq. (16) can accurately predict the results within an interval of $\pm 26 \%$; however, it was able to predict $90 \%$ of the results within $\pm 10 \%$. The correlation is presented as a means of concisely capturing the behavior observed in the parametric space considered and can be used in "delta-mode" to quickly establish the enhancement or degradation in heat transfer that will occur for a given bank of jets relative to being centered directly under the cylinder axis at a particular axial and vertical spacing. Without experimental verification, it is not recommended that the correlation be used to predict the absolute convective heat transfer from the cylinder.

\section{Conclusions}

A CFD study has been carried out to study the convective heat transfer due to multiple circular jets impinging on an isothermal horizontal cylinder. The unique aspect of the study is the large jet-to-cylinder diameters considered $(0.15 \leqslant d / D \leqslant 0.31)$, and the close proximity of the jet exit to the cylinder. The results were computed using the commercial software FLUENT ${ }^{\mathrm{TM}}$ over a parametric space that is relevant to the cement production industry. The following summary points are made:

1. As $R e_{d}$ gets smaller, variations in non-dimensional parameters have less of an impact on changes in the average Nusselt number of the horizontal cylinder.
2. For a centered jet $(x / d=0)$, when $d / D \geqslant 0.23$ and $y / d$ increases, the average Nusselt number decreases for all $z / d$ parameters studied. This same trend is seen for the offset cases in this range of dimensionless jet diameter.

3. When $d / D=0.15$, increasing $y / d$ results in a larger average Nusselt number for a centered jet at $z / d=1.6$ and 1.8 . For a fullyoffset jet with the same parameters, the same behavior is observed, although with smaller increases.

4. For $d / D=0.15$ offsetting the jets results in a larger average Nusselt number at certain axial spacings; i.e., when $y / d=0.4$, for $z$ $d=1.4-2.4$, and when $y / d=2.1$ for $z / d \geqslant 1.4$, the average Nusselt number is larger for an offset jet.

5. The average Nusselt number does not follow a specific trend as $z / d$ increases, but rather it is subject to regions of increase and decrease, depending on other parameters. Beyond $z / d 2.9$, a constant value of average Nusselt number is reached due to low jetinterference from neighboring jets.

\section{Acknowledgments}

The authors gratefully acknowledge the financial support from Mitacs Canada.

\section{References}

[1] K. Mujumdar, V. Ranade, Simulation of rotary cement kiln using a onedimensional model, Chem. Eng. Res. Des. 84 (2006) 165-177.

[2] E. Mastorakos, A. Massais, C.D. Tsakiroglou, D.A. Goussis, V.N. Burganos, A.C Payatakes, CFD predictions for cement kilns including flame modelling, heat transfer and clinker chemistry, Appl. Math. Model. 23 (1) (1999) 55-76.

[3] H. Martin, Heat and mass transfer between impinging gas jet and solid surfaces, Adv. Heat Transfer 13 (1977) 1-60.

[4] K. Jambunathan, E. Lai, M.A. Moss, B.L. Button, A review of heat transfer data for single circular jet impingement, Int. J. Heat Fluid Flow 13 (1992) $106-$ 115.

[5] N. Zuckerman, N. Lior, Jet impingement heat transfer: physics, correlations, and numerical modeling, Adv. Heat Transfer 39 (2006) 565-631.

[6] A.J. Yule, Large-scale structure in the mixing layer of a round jet, J. Fluid Mech. 89 (1978) 413-432.

[7] R. Gardon, J.C. Akfirat, The role of turbulence in determining the heattransfer characteristics of impinging jets, Int. J. Heat Mass Transf. 8 (1965) 1261-1272.

[8] E.E.M. Olsson, L.M. Ahrne, A.C. Tragardh, Heat transfer from a slot air jet impinging on a circular cylinder, J. Food Eng. 63 (2004) 393-401.

[9] E.E.M. Olsson, L.M. Ahrne, A.C. Tragardh, Flow and heat transfer from multiple slot air jets impinging on circular cylinders, J. Food Eng. 67 (2005 273-280.

[10] T.-M. Jeng, S.-C. Tzeng, R. Xu, Heat transfer characteristics of a rotating cylinder with lateral air impinging jet, Int. J. Heat Mass Transf. 70 (2014) 235249

[11] C.S. McDaniel, B.W. Webb, Slot jet impingement heat transfer from circular cylinders, Int. J. Heat Mass Transf. 43 (2000) 1975-1985.

[12] F. Gori, L. Bossi, On the cooling effect of an air jet along the surface of a cylinder, Int. Commun. Heat Mass Transfer 27 (2000) 667-676.

[13] F. Gori, L. Bossi, Optimal slot height in the jet cooling of a circular cylinder, Appl. Therm. Eng. 23 (2003) 859-870.

[14] E.M. Sparrow, A. Alhomoud, Impingement heat transfer at a circular cylinder due to an offset or non-offset slot jet, Int. J. Heat Mass Transf. 27 (1984) 2297 2306 .

[15] S.A. Nada, Slot/slots air jet impinging cooling of a cylinder for different jetscylinder configurations, Heat Mass Transf. 43 (2006) 135-148.

[16] N. Zuckerman, N. Lior, Radial slot jet impingement flow and heat transfer on a cylindrical target, J. Thermophys. Heat Transfer 21 (2007) 548-561.

[17] A.A. Tawfek, Heat transfer due to a round jet impinging normal to a circular cylinder, Heat Mass Transf. 35 (1999) 327-333.

[18] D.H. Lee, Y.S. Chung, D.S. Kim, Turbulent flow and heat transfer measurements on a curved surface with a fully developed round impinging jet, Int. J. Heat Fluid Flow 18 (1997) 160-169.

[19] M. Kumada, I. Mabuchi, Y. Kawashima, Mass transfer on a cylinder in the potential core region of a two-dimensional jet, Heat Transfer - Jpn. Res. 2 (1973) 53-66.

[20] X.L. Wang, D. Motala, T.J. Lu, S.J. Song, T. Kim, Heat transfer of a circular impinging jet on a circular cylinder in crossflow, Int. J. Therm. Sci. 78 (2014) $1-8$.

[21] X.L. Wang, H.B. Yan, T.J. Lu, S.J. Song, T. Kim, Heat transfer characteristics of an inclined impinging jet on a curved surface in crossflow, J. Heat Transfer 136 (2014) 81702. 
[22] E.M. Sparrow, C.A.C. Altemani, A. Chaboki, Jet-impingement heat transfer for a circular jet impinging in crossflow on a cylinder, Trans. ASME J. Heat Transfer 106 (1984) 570-577.

[23] D. Singh, B. Premachandran, S. Kohli, Experimental and numerical investigation of jet impingement cooling of a circular cylinder, Int. J. Hea Mass Transf. 60 (2013) 672-688.
[24] L. Dong, C. Leung, C. Cheung, Heat transfer of a row three butane/air flame jets on a flat plate, Int. J. Heat Mass Transf. 46 (2003) 113-125.

[25] ANSYS Inc, ANSYS FLUENT 14.5 Theory Guide, 2012.

[26] Fluent Inc., FlowLab 1.2 Flow Over a Cylinder, 2005. 